\title{
Large-area, kirigami topology structure-induced highly stretchable and flexible interconnects: Directly printing preparation and mechanic mechanism
}

\author{
Xuan $\mathrm{Li}^{1,3 \dagger}$, Xiaoli Ruan ${ }^{2 \dagger}$, Weijing Yao ${ }^{1}$, Li Liu ${ }^{1}$, Bin Tian ${ }^{1}$, Huanjun Wang ${ }^{1}$, Yu Feng ${ }^{1}$, Re Xia ${ }^{2^{*}}$ and \\ $\mathrm{Wei} \mathrm{Wu}^{1,3^{*}}$
}

\begin{abstract}
Integrating the topology design and printing method offers a promising methodology to realize large stretchability for interconnects. Herein, eco-friendly and waterbased Ag nanowires (NWs) inks were formulated and used for screen-printing highly stretchable and flexible interconnects on a large area (more than $335 \mathrm{~mm} \times 175 \mathrm{~mm}$ ). The stretchability of the interconnects was realized by introducing kirigami topology structures. The topology designed models were established to simulate the influence of kirigami patterns on wire compliance and to estimate the maximum stretchability via finite element analysis (FEA). The mechanic mechanism results demonstrate that an increase of the wave numbers results in larger stretchability, and the rectangular type of wave shows better stretchability than the zigzag and sine structures. Comparatively, the electrical and mechanical properties of the interconnects were measured and analyzed, and the experimental results were consistent with FEA. The electric conductivity of the interconnects is stable at $\sim 10,427 \mathrm{~S} \mathrm{~cm}^{-1}$ even after 1000 cycles of $15.83 \mathrm{~mm}$ radius bending, $280 \%$ stretching and $200 \%$ twisting-stretching deformation, demonstrating outstanding mechanical reliability of the interconnects. The topology designed interconnects have been applied in stretchable flexible light-emitting diode, indicating their broad application prospects in next-generation stretchable electronics.
\end{abstract}

Keywords: screen printing, stretchable and flexible conductors, finite element analysis, printed electronics

\section{INTRODUCTION}

In recent years, various types of electronic products have become increasingly popular with the continuous development of electronic science and technology. Additionally, stretchable and flexible electronics have drawn increasing attention in the industry, because the stretchability will significantly expand the application range of electronics [1-3]. Stretchable and flexible conductors are the key to fabricate next-generation stretchable electronics such as stretchable light-emitting diodes (LEDs) $[4,5]$, stretchable displays $[6,7]$, electronic skin $[8,9]$, strain sensors [10-14], electromagnetic interference (EMI) shielding absorbers [15-17] and gun propellant [18].

The greatest challenge in preparing stretchable conductors is to achieve stretchability and keep them stable under deformation conditions. In general, the stretchability of conductors can be achieved by two methods, including the introduction of intrinsic stretchable materials and specific stretchable structures [19]. For example, in our previous work, highly conductive stretchable interconnects were prepared by using fractal silver particles and polydimethylsiloxane, and the conductors can be bent, stretched to $100 \%$ and twisted up to $180^{\circ}$ [20]. Moreover, employing specific stretchable structure has drawn increasing attention because of excellent stretchability [21-25]. For example, Wiley and co-workers [26] reported stretchable conductive composites by using $\mathrm{Cu}$ Ag nanowires (NWs). A serpentine structure prepared by vacuum filtration was introduced and these stretchable composites had a conductivity of $1000 \mathrm{~S} \mathrm{~cm}^{-1}$ at initial and $10 \mathrm{~S} \mathrm{~cm}^{-1}$ when strained to $300 \%$. Although the in-

\footnotetext{
${ }^{1}$ Laboratory of Printable Functional Nanomaterials and Printed Electronics, School of Printing and Packaging, Wuhan University, Wuhan 430072, China

${ }^{2}$ Key Laboratory of Hydraulic Machinery Transients, School of Power and Mechanical Engineering, Wuhan University, Wuhan 430072, China

${ }^{3}$ Shenzhen Research Institute of Wuhan University, Shenzhen 518057, China

These authors contributed equally.

* Corresponding authors (emails: weiwu@whu.edu.cn (Wu W); xiare@whu.edu.cn (Xia R))
} 
troduction of specific stretchable structures in that work made it possible to achieve large stretchability, its conductivity sharply decreased to $1 \%$ when stretched to $300 \%$, and its principle of achieving stretchability need to be further studied.

Recently, researchers have studied the topology designs or fractal structures and used the vacuum filtration, photolithography method or laser milling to realize the fabrication of stretchable interconnects. For example, Rogers and Huang's group used photolithography [27] and laser milling method [28] to fabricate stretchable interconnects. However, these methods are high-cost and hard to realize versatile pattern designs. Consequently, it is expected that new manufacturing process should be developed to overcome these shortcomings. Printing technology is the exactly one that is both low-cost and facile to design versatile patterns [29], which potentially spawned a lot of applications, such as LEDs [30,31], thinfilm transistors (TFTs) [32,33], sensors [34,35], supercapacitors [36,37], solar cells [38,39], heaters [40,41], and anti-counterfeiting [42]. The main methods for printing electronics include inkjet printing, roll-to-roll printing and screen printing $[1,43]$. Compared with other printing methods, screen printing technology is simple, low-cost and suitable for large-area application.

A key problem for manufacturing large-area screenprinted stretchable flexible interconnects is to formulate uncomplicated and high-yield material-based inks. Although various inks have been developed, such as $\mathrm{Ag}$ flakes [44], and $\mathrm{Ag} / \mathrm{AgCl}$ [45], their preparation processes are complicated, and these reported stretchable flexible conductors either have low electric conductivity or lose remarkable conductivity under large stretching deformation. Comparatively, Ag NWs are a perfect candidate due to its high conductivity and flexibility, and their simple preparation process is suitable for massproduction. However, it is a challenge to develop printed stretchable and flexible conductors with Ag NWs ink because of the difficulty to prepare large-scale uniform Ag NWs and hinder the agglomeration and precipitation.

Herein, Ag NWs were synthesized by a modified solvothermal method to formulate the ink. The printability of the ink was investigated by studying the rheological behavior and viscoelasticity. Then the stretchable interconnects were fabricated by the combination of direct screen printing and kirigami method. The effects of different topology structures and wave numbers on stretchability were investigated by building corresponding models via finite element analysis (FEA). On the other side, the electrical and mechanical properties were further investigated by electrical tensile experiments, which showed great consistence with the FEA results. The optimal interconnect shows a high electrical conductivity of $10,427 \mathrm{~S} \mathrm{~cm}^{-1}$ and good stability under 1000 cycles of bending, stretching and twisting-stretching.

\section{EXPERIMENTAL SECTION}

\section{Materials}

$\mathrm{AgNO}_{3}$ (AR), ethyl alcohol $\left(\mathrm{C}_{2} \mathrm{H}_{5} \mathrm{OH}, \mathrm{AR}\right)$ and ethylene glycol (EG, 98\%) were obtained from Sinopharm Chemical Reagent Co., Ltd. $\mathrm{CuCl}_{2} \cdot 2 \mathrm{H}_{2} \mathrm{O}$ (AR) was obtained from Shanghai Aladdin Reagents Co., Ltd. Polyvinylpyrrolidone (PVP, molecular weight of $\sim 360,000$ ) was obtained from Sigma-Aldrich.

\section{Synthesis of Ag NWs}

Ag NWs were synthesized by a modified solvothermal method. Firstly, PVP $(1.665 \mathrm{~g})$ and $\mathrm{CuCl}_{2}(0.0019 \mathrm{~g})$ were dispersed in $100 \mathrm{~mL}$ of EG. $\mathrm{AgNO}_{3}(1.7 \mathrm{~g})$ was dissolved in $100 \mathrm{~mL}$ of EG. Then, the PVP-CuCl${ }_{2}$ solution was added to the $\mathrm{AgNO}_{3}$ solution dropwise. The mixed solution was transferred to a $250 \mathrm{~mL}$ autoclave and heated at $160^{\circ} \mathrm{C}$ for $4 \mathrm{~h}$. The Ag NWs were washed for several times by using ethanol and water. Finally, the products were dissolved in ethanol for the formulation of Ag NWs ink.

\section{Formulation of Ag NWs ink for screen printing}

The Ag NWs ink was formulated by mixing Ag NWs with PVP. Firstly, PVP $(0.01 \mathrm{~mol})$ was dissolved in $5 \mathrm{~mL}$ of ethanol and $5 \mathrm{~mL}$ of deionized water to form the additive solution. Then, $1 \mathrm{~g}$ of $\mathrm{Ag}$ NWs solution (10.56 wt\% in ethanol) and $0.4 \mathrm{~mL}$ of $1 \mathrm{~mol} \mathrm{~L}^{-1}$ PVP additive solution were mixed, and then sonicated and stirred for $5 \mathrm{~min}$ to formulate the Ag NWs ink.

\section{Fabrication of the stretchable and flexible Ag NWs interconnects}

The stretchable and flexible interconnects were fabricated by screen printing and kirigami method. Firstly, The polyester (PET) film was cleaned by ethanol and deionized water. Next, Ag NWs ink was printed on PET using a 300 mesh counts screen plate with multifarious patterns. Then the printed PET was sintered at $120^{\circ} \mathrm{C}$ for $30 \mathrm{~min}$ to remove the solvent and reduce the resistance. After being cooled to room temperature, the patterned PET was cut along the pattern to gain the stretchable and flexible interconnects. 


\section{Characterizations}

The morphologies of Ag NWs and printed tracks were characterized by scanning electron microscopy (SEM, Hitachi S-4800). The sheet resistance of the stretchable and flexible interconnects was measured at room temperature by using a four-point probe resistance tester (FP-001). The rheological behavior and oscillating rheological behavior of the Ag NWs ink were studied using a Kinexus pro rotational rheometer. The stretchable and flexible interconnects were fixed on a customized stage to study the real-time sheet resistance under bending and stretching state using a Keithley 2400 source meter. The uniaxial tensile mechanical characteristics of the varied layouts were exploited via FEA performed with ABA$\mathrm{QUS}^{\circledR}$ standard.

\section{RESULTS AND DISCUSSION}

A schematic diagram of the fabrication of stretchable flexible interconnects is demonstrated in Scheme 1. Firstly, the Ag NWs were synthesized by a modified solvothermal method, in which the volume of the solution was 10 times that of our previous work [46]. After being washed several times, the water-based screen printing ink was prepared by combining the as-prepared Ag NWs solution with PVP binder. Most of the stretchable substrates, such as fabric and rubber, have poor printability; in other word, it is hard or complex to print versatile patterns on stretchable substrates. Consequently, conventional PET substrate was selected here because it is easy to be printed on and frequently-used in electron industry. A 300-mesh screen plate with customized patterns was used to screen-print the ink onto the PET substrate. Then the screen-printed PET was sintered at $120^{\circ} \mathrm{C}$ for $30 \mathrm{~min}$. The final stretchable and flexible interconnects were obtained by cutting the screen-printed PET along the pattern with traditional kirigami method.

Fig. 1a shows that the prepared Ag NWs have a uniform morphology with an average diameter of $141.53 \mathrm{~nm}$ and a length of $39.96 \mu \mathrm{m}$, which is beneficial to form a good conductive network structure. The formulated Ag NWs ink consists of three elements, the Ag NWs as the conductive components, PVP as the binder, and water and ethanol serving as the solvent. The printability of the ink was investigated by studying the rheological behavior and viscoelasticity. As shown in Fig. 1b, there are three periods in this test, the shear rate in the first period of $20 \mathrm{~s}$ is $0.1 \mathrm{~s}^{-1}$ before printing; the shear rate in the second period of $30 \mathrm{~s}$ is $200 \mathrm{~s}^{-1}$ to simulate the squeegee stroke; finally the shear rate decreases to $0.1 \mathrm{~s}^{-1}$ for $100 \mathrm{~s}$ to simulate the viscosity recovery of the ink after being transferred to PET. The viscosity is stable at around 94.51 Pa s when the shear rate is $0.1 \mathrm{~s}^{-1}$. As the shear rate increases to $200 \mathrm{~s}^{-1}$, it decreases sharply to $0.5742 \mathrm{~Pa} \mathrm{~s}$, which ensures the ink to be transferred through the mesh to the PET during scraping. Meanwhile, low viscosity improves the definition of printed lines. After finishing

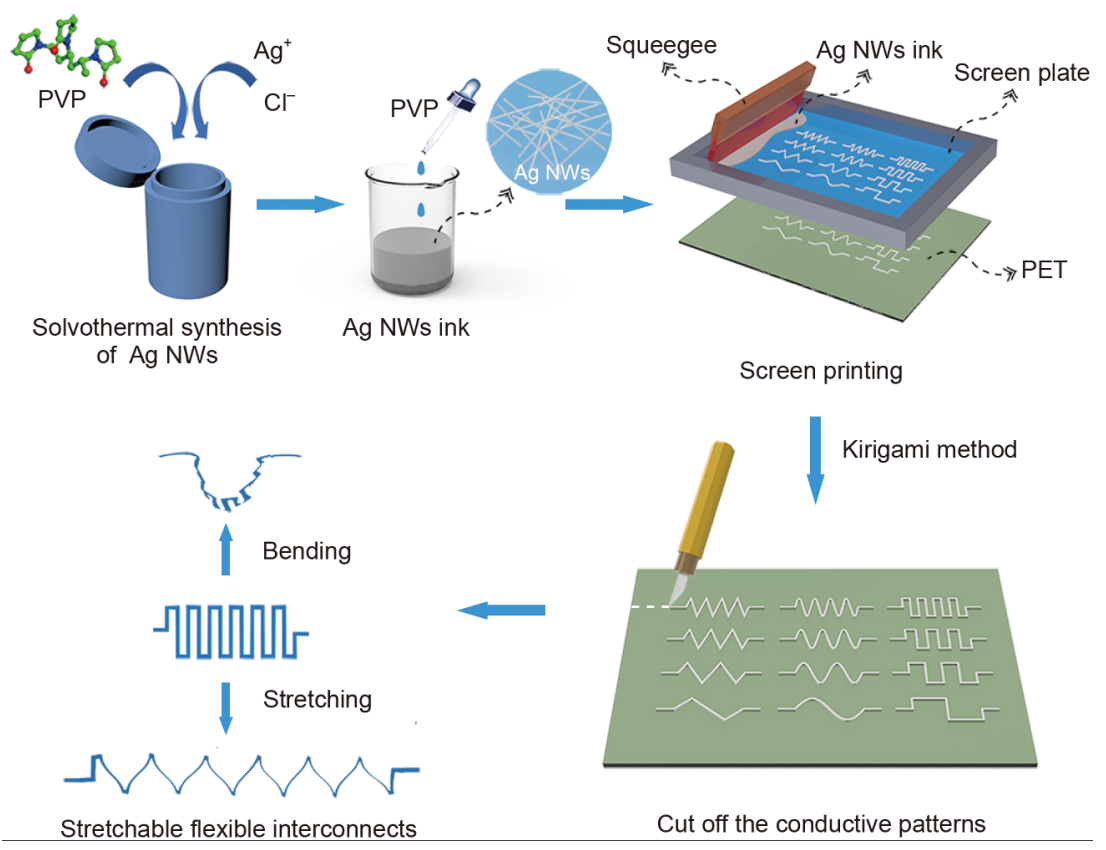

Scheme 1 Schematic diagram of the fabrication of stretchable flexible interconnects. 

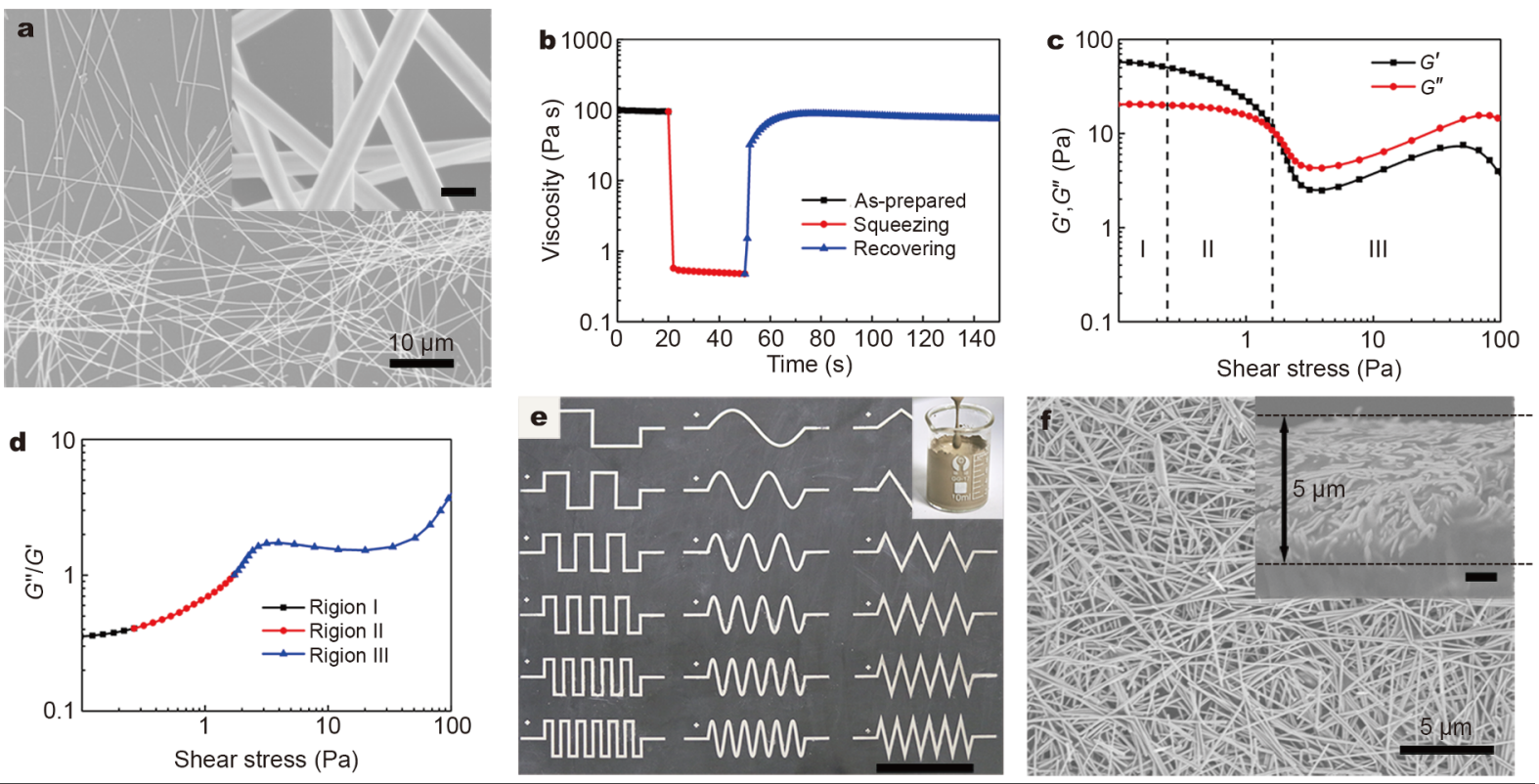

Figure 1 (a) Low-magnification SEM image of the Ag NWs (the inset is high-magnification SEM image of the Ag NWs; the scale bar is $200 \mathrm{~nm}$ ). (b) Rheological behavior of the ink. (c) Change of $G^{\prime}$ and $G^{\prime \prime}$ as shear stress. (d) Variation of $G^{\prime \prime} / G^{\prime}$ with shear stress. (e) Optical photography of the screenprinted PET (the inset is an optical photography of as-prepared Ag NWs ink; the scale bar is $5 \mathrm{~cm}$ ). (f) SEM image of the surface of the printed PET (the inset is the cross-section SEM image of the printed PET; the scale bar is $1 \mu \mathrm{m}$ ).

scraping, the viscosity returns to 88.89 Pa s at $78 \mathrm{~s}$ and the percentage of recovery is over $94 \%$, demonstrating good elasticity of the ink, which is attributed to the uniform morphology and network structures of Ag NWs. In this process, the ink can be uniformly formed into a film after screen printing.

Furthermore, the viscoelasticity of the ink was studied by an oscillating rheological measurement. Fig. 1c demonstrates the change of storage modulus $\left(G^{\prime}\right)$ and the loss modulus $\left(G^{\prime \prime}\right)$ of the ink. The change of $G^{\prime \prime} / G^{\prime}$ ratio as a function of shear stress is demonstrated in Fig. 1d. As shown in Fig. 1c, there are three regions: firstly, a compact structure between Ag NWs is formed. Therefore, the structure of the ink is not damaged by external shear stress in this region. At this time, the ratio of $G^{\prime \prime} / G^{\prime}$ is about 0.41 (Fig. 1d). In the second region, $G^{\prime}$ and $G^{\prime \prime}$ decrease as the shear stress increases, indicating the structure of the ink is gradually destroyed. However, the storage modulus is still greater than the loss modulus, and the ink still exhibits more solid behavior. The third region starts from the storage modulus equal to the loss modulus (shear stress is about 1.735 Pa). As the shear stress further increases, the loss modulus is gradually larger than the storage modulus, and the ink behavior gradually flows to the fluid. If the corresponding shear stress at the junction is too large, the ink will stick to the plate and block the mesh. Fig. 1d shows the change of $G^{\prime \prime} / G^{\prime}$ as a function of shear stress. The smaller the ratio of $G^{\prime \prime} / G^{\prime}$, the larger the viscosity of the ink, and the more difficult it is to pass through the screen. An excessive $G^{\prime \prime} / G^{\prime}$ ratio means that the viscosity of the ink is too small, and it is easily diffused after passing through the screen mesh, resulting in a decline in the printing accuracy. As presented in Fig. 1e, owing to the good printability of ink, a distinct image is printed over a large area $(335 \mathrm{~mm} \times 175 \mathrm{~mm})$ on PET. The inset in Fig. 1e is a typical photograph of the asprepared Ag NWs ink. In addition, the optical photograph of the screen printer, just printed PET and curly full-printed flexible PET are presented in Fig. S1 (see Supplementary information). The top view SEM image of the printed tracks is shown in Fig. 1f, and the homogeneous distribution of Ag NWs reveals compact connection of Ag NWs after sintering treatment, which is crucial to forming a good conductive path. As shown in the inset of Fig. 1f, the cross-section view SEM image shows that the thickness of Ag NWs layer is about $5 \mu \mathrm{m}$. The low sheet resistance $(0.19 \Omega / \mathrm{sq})$ results from the uniform high aspect ratio Ag NWs and the dense interconnected Ag NWs.

Different topology arrangements are considered as an important factor influencing the wire compliance. By varying the wave numbers and wave types, the stretch- 
ability of the pre-designed layouts was characterized by the uniaxial tensile simulations via FEA. Detailed analyses of the effects of these geometric parameters on mechanical stretchability are provided. Three distinct layouts were investigated, each of which was arranged periodically with different number of waves. The cross-section is assumed to be identical in all defined patterns, and it has the width of $1.5 \mathrm{~mm}$ and thickness of $0.15 \mathrm{~mm}$. Rectangular and zigzag wave layouts were constrained by overall scale, keeping the number of waves changed only. The sine wave layout was controlled by the equation as follows:

$x=48.5 t$,

$y=9.25 \sin (360 t \cdot N)$,

where $N$ is varied from 1 to 6 correspondingly $(0 \leq t \leq 1)$. During the tensile simulation along the $x$ direction, all structures were considered to be free-standing to focus on the mechanical properties of the wave defined patterns. For a more accurate prediction of the stretchability of the varied kirigami topologies, one end of the wire-like structure was fixed initially while four degrees of freedom were remained for the other end leaving the folding wave in the middle free to be stretched without severe out-ofplane wrinkling deformations. The tensile-bending cou- pled deformations of the configuration as a result of increasing displacement loads were measured. Meanwhile, the strains of the layouts were monitored when the limit strain of the parent material was reached, which meant the stretch deformation was about to break in any section. Based on the advancing front algorithm, the patterned wires were meshed using three-dimensional solid models (C3D8I). Four different mesh densities were analyzed in case of the rectangular wave: mesh-1 with 4695 elements, mesh-2 with 6768 elements, mesh-3 with 11,352 elements and mesh- 4 with 18,810 elements in the assembly (mesh size was $0.30,0.25,0.20$, and $0.15 \mathrm{~mm}$, respectively). While the element size of $0.15 \mathrm{~mm}$ was used for all the tensile simulations in this study, adequately enough for the comparative investigation of the geometry effect on the tensile mechanical behaviors.

Fig. 2 shows the deformed configurations and stress distribution of the rectangular wave in response to the maximum strain applied at varied wave numbers. With the increase of the wave number, the rectangular type of wave layout achieves a higher ultimate strain $(\varepsilon)$ as marked in Fig. 2. All of the wires break before being stretched into straight and the final degree of straightness depends on the wave number. A symmetry stress distribution is observed in each of the layout, and thus we can focus on the representative pattern including the half

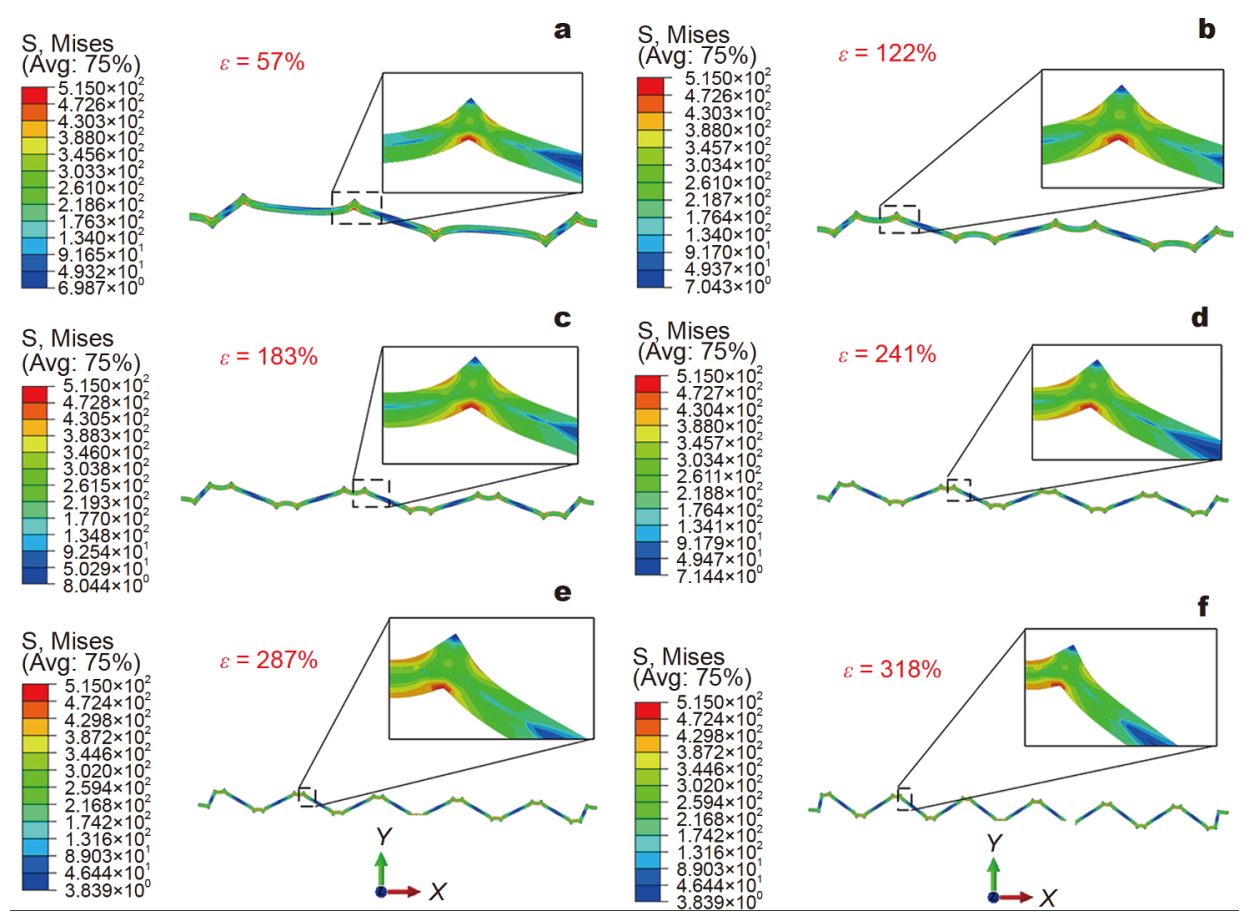

Figure 2 Von Mises stress distribution of the deformed rectangular layouts with varied wave numbers: (a) one, (b) two, (c) three, (d) four, (e) five, and (f) six. 
of parallel part (left) and the half of adjacent perpendicular part (right), as shown in the drawing of partial enlargement (see the drawing inside the frame in Fig. 2). It is found that the maximum stress locates around the inner corner and the periphery of external corner, which predicts the first break location of the structure. The deformed layouts also indicate that the part parallel to the tensile direction endures higher stress than the perpendicular one as the larger area of blue contour are observed in perpendicular part. Both the sine and zigzag type wave of layouts show similar tendency, except that the stress distribution is symmetry in left and right part of each layout. This results from the symmetry of the structure as illustrated in Figs S2 and S3. For the comparison among three wave types, eighteen finite element models were constructed (summarized in Fig. 3a for analysis. In case of two wave number of the layouts, the geometries before and after deformation are presented in Fig. 3b-d. It can be seen that the rectangular type of wave shows the best stretchability, while the zigzag wave is relatively inferior. Despite the constraint over the $z$-direction, out-of-plane displacement is observed in the overlap region (for example, as pointed out in Fig. $3 \mathrm{~b}$ ) predicting the strong tendency of out-of-plane deformation in experimental tests.

The ultimate strains obtained from FEA are displayed in Fig. 4a for the three configurations as a function of wave number. We can conclude that these distinct layouts affect the stretchability via the degree of folding complexity. An increase of the wave number in the layout results in the improvement of the ability to bear greater stretch strain. Meanwhile, the final deformed structure deviates more severely from the straight line (see Fig. 2, Figs S2 and S3). The rectangular type of wave wires is found to have the greatest stretchability, followed by sine wave and zigzag wave wires.

After we investigated the influence of topology designs on the maximum stretchability via theoretical FEA calculations, we used printing method and kirigami method to apply the topology design to the electrode. We further investigated the mechanical reliability of the interconnects based on three kinds of basic structures by experimental tests. The relative resistance changes of interconnects under stretching state are present in Fig. 5. The photographs of typical samples based on 3 kinds of topology structures are presented in Fig. S4, which illustrates that these interconnects have a high resolution. The resistances of different printed interconnects with same geometry are similar; for instance, the average resistance of the interconnects with 1 zigzag is $7.23 \Omega$. As shown in Fig. 5 , the tensile limits of interconnects based on zigzag increase from $17 \%$ to $209 \%$ with increasing number of waves; the tensile limits of interconnects based on sine wave increase from $18 \%$ to $271 \%$ with increasing number of waves. Similarly, the tensile limits of interconnects based on rectangular wave increase from 59\% to 302\% with increasing number of waves. It is concluded that tensile limit increases with increasing number of basic structures. Furthermore, the tensile limit of interconnects based on rectangular waves is always the highest. The

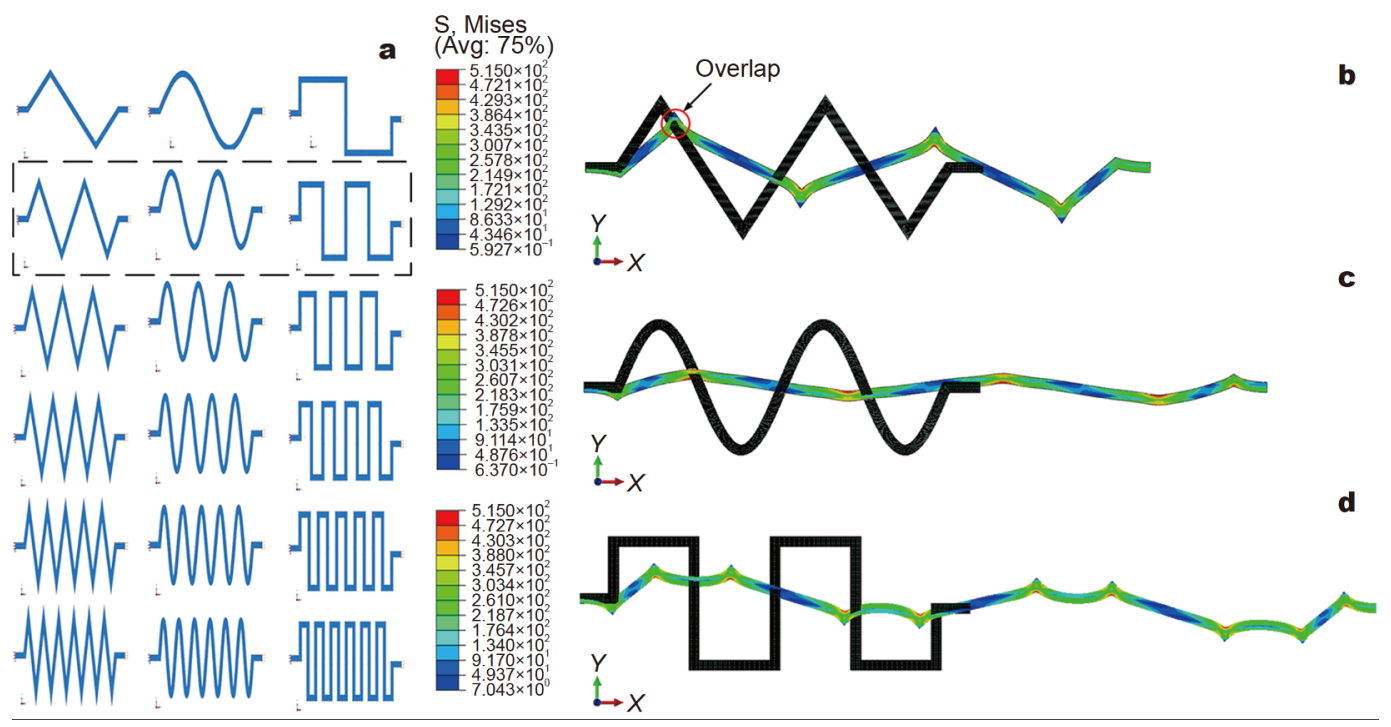

Figure 3 (a) Three pre-designed layouts and corresponding parameterized structures; the stress distribution on structures before and after deformation: (b) zigzag wave; (c) sine wave; (d) rectangular wave (only two waves of each type are presented here). 

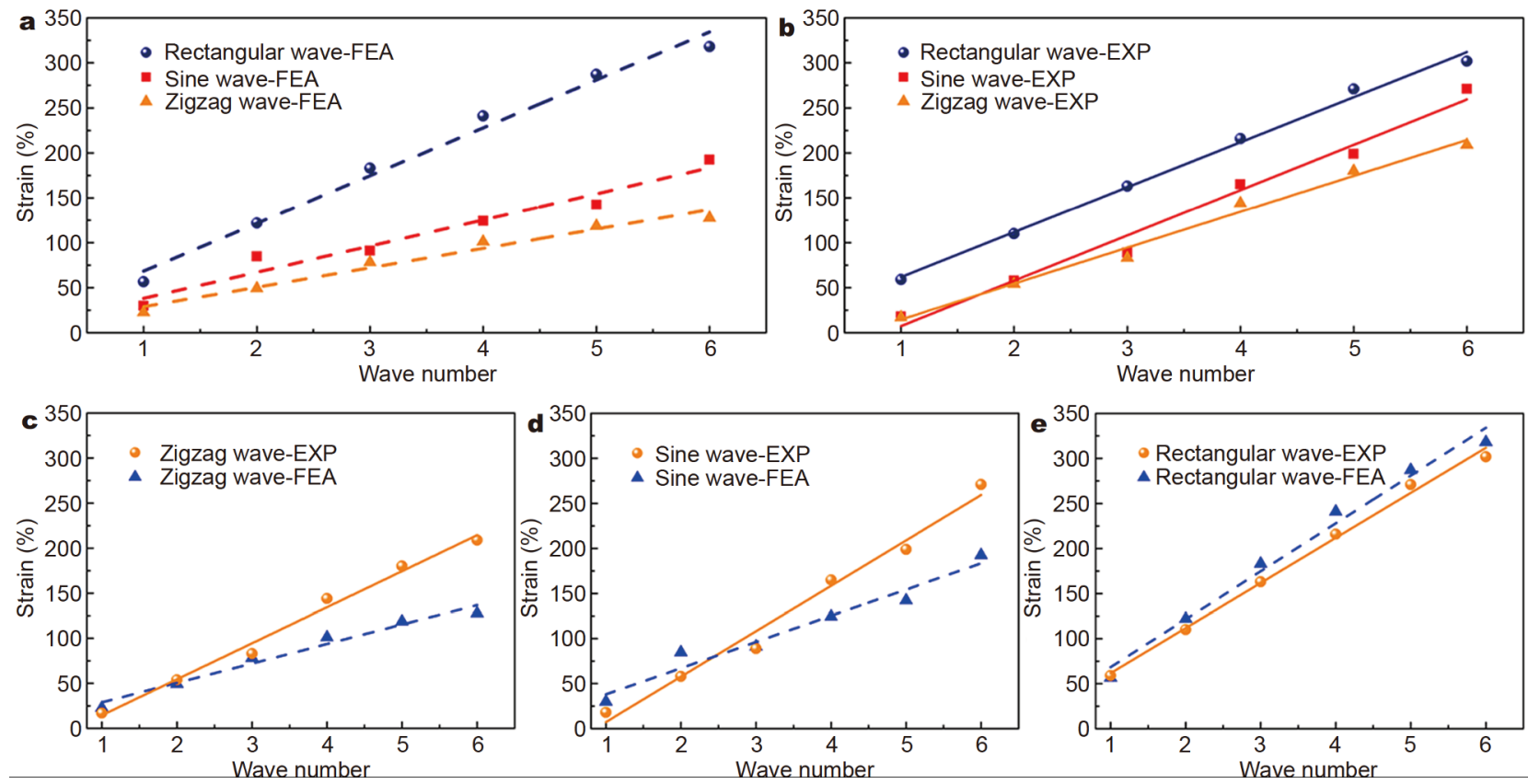

Figure 4 Stretchability obtained from (a) FEA and (b) experiments (EXP). A separate comparison between FEA and experimental results of the (c) rectangular wave, (d) sine wave and (e) zigzag wave.

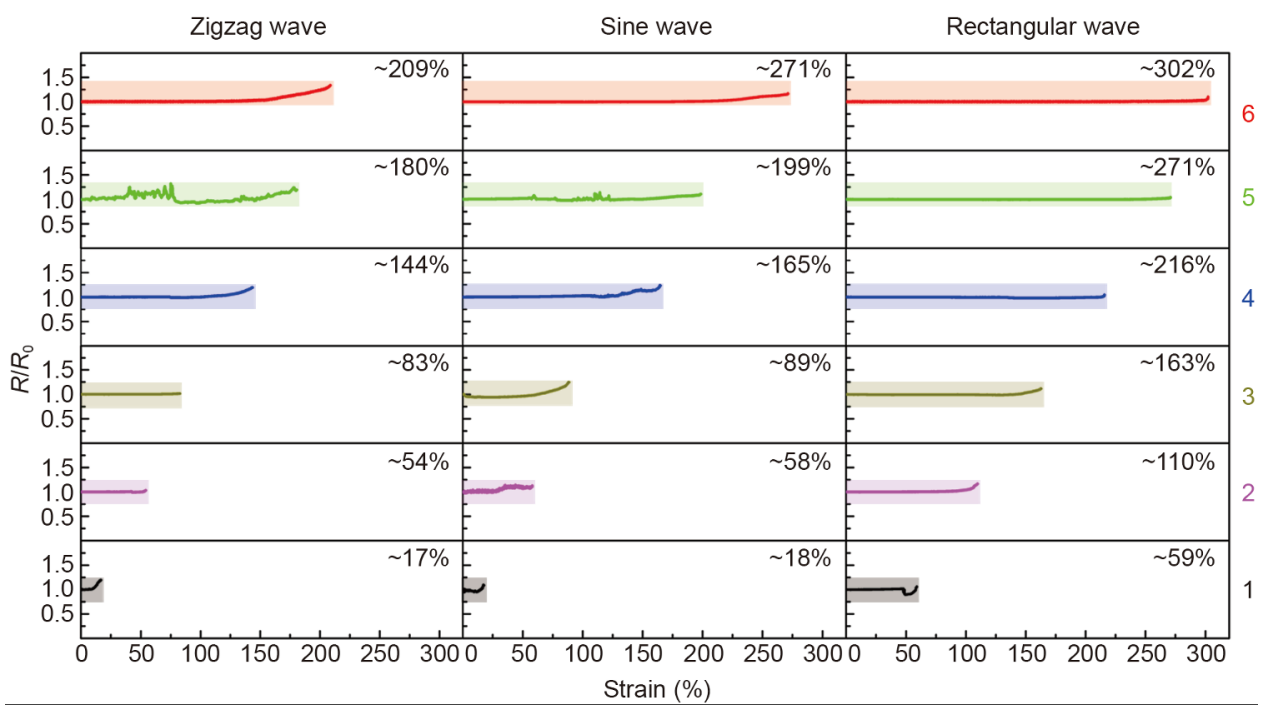

Figure 5 Relative resistance changes of interconnects based on three kinds of topology structures with 1-6 waves under stretching states. All the samples have the same size of $60 \mathrm{~mm}$ long and $20 \mathrm{~mm}$ wide.

interconnect with 6 rectangular waves has the highest tensile limit of $302 \%$.

Comparisons between FEA and experimental tests are quantified in Fig. 4. The solid lines denote the experimental results and the dashed lines represent the FEA results. Excellent agreement between the experimental and numerical approaches is observed, and specially, the rectangular type of wave layout possesses much better stretchability than the other two layouts. Fig. 4c-e respectively show the calculated tensile strains of zigzag wave, sine wave, and rectangular wave layouts for both numerical analysis and experiments, and it can be seen that acceptable differences exist mainly in the sine wave and zigzag wave layouts. For the experimental investiga- 
tions, ultimate strain was calculated till the whole section was broken and a perfect corner condition between disoriented wires cannot be manufactured, while an ideal sharp corner was adopted in FEA model. Otherwise, it shows great consistence with the experimental test when viewed from the overall trend, which verifies the feasibility of the novel manufacturing process.

Fig. 6a presents the results of the relative resistance of the optimal interconnects as a function of bending cycles. The bending radius is $15.83 \mathrm{~mm}$, and the bending and release time is $1 \mathrm{~s}$. The resistance of interconnects remains almost unchanged after 1000 bending-releasing cycles. Fig. $6 \mathrm{~b}$ presents the relative resistance under stretching cycles, the strain of stretching cycle is $280 \%$, and the speed and duration of each stretching and release are $1.68 \mathrm{~cm} \mathrm{~s}^{-1}$ and $10 \mathrm{~s}$, respectively. The resistance of interconnects remains almost unchanged after 1000 stretching cycles. Fig. $6 \mathrm{c}$ presents the relative resistance under the twisting-stretching. The strain of twistingstretching cycle is $200 \%$, the angle of twist is $180^{\circ}$, the speed and duration of each twisting-stretching and release are $1.20 \mathrm{~cm} \mathrm{~s}^{-1}$ and $10 \mathrm{~s}$, respectively. The resistance of interconnects remains almost unchanged after 1000 stretching-releasing cycles. In addition, the adhesion of the Ag NWs ink pattern was tested and the results are shown in Fig. S5. It demonstrates that the resistance of the interconnects is stable after 20 tape test cycles. Furthermore, the relative change of resistance is only $0.3 \%$ after 165 days, demonstrating excellent air stability of the Ag NWs patterns. As shown in Fig. 6d, for the previously reported stretchable interconnects, their conductivity is either low, or the stretch rate is very low, or it declines sharply under stretching state [26, 47-49]. Comparatively, the interconnects in our work possess outstanding performance in three aspects: conductivity, stretchability, and the stability at large stretching state.

The outstanding electromechanical performance can be proved by simple circuits. As shown in Fig. 7, the LED is lit after applying the voltage of $6 \mathrm{~V}$ to the interconnects. The brightness of the LED remains unchanged even if the interconnects are under bending, stretching, twisting and twisting-stretching states. Fig. S6 and video S1 (in Supplementary information) present the stretchable LED circuit with 6 units of rectangular wave at different percentage of strain, illustrating the brightness of the LED is stable during $280 \%$ stretching and releasing. In addition, a stretchable flexible LED circuit was attached to a knee (see Fig. S7), and its brightness is stable after bending and stretching. Similarly, it can be applied to other joints of body or robots, which proves its broad application pro-
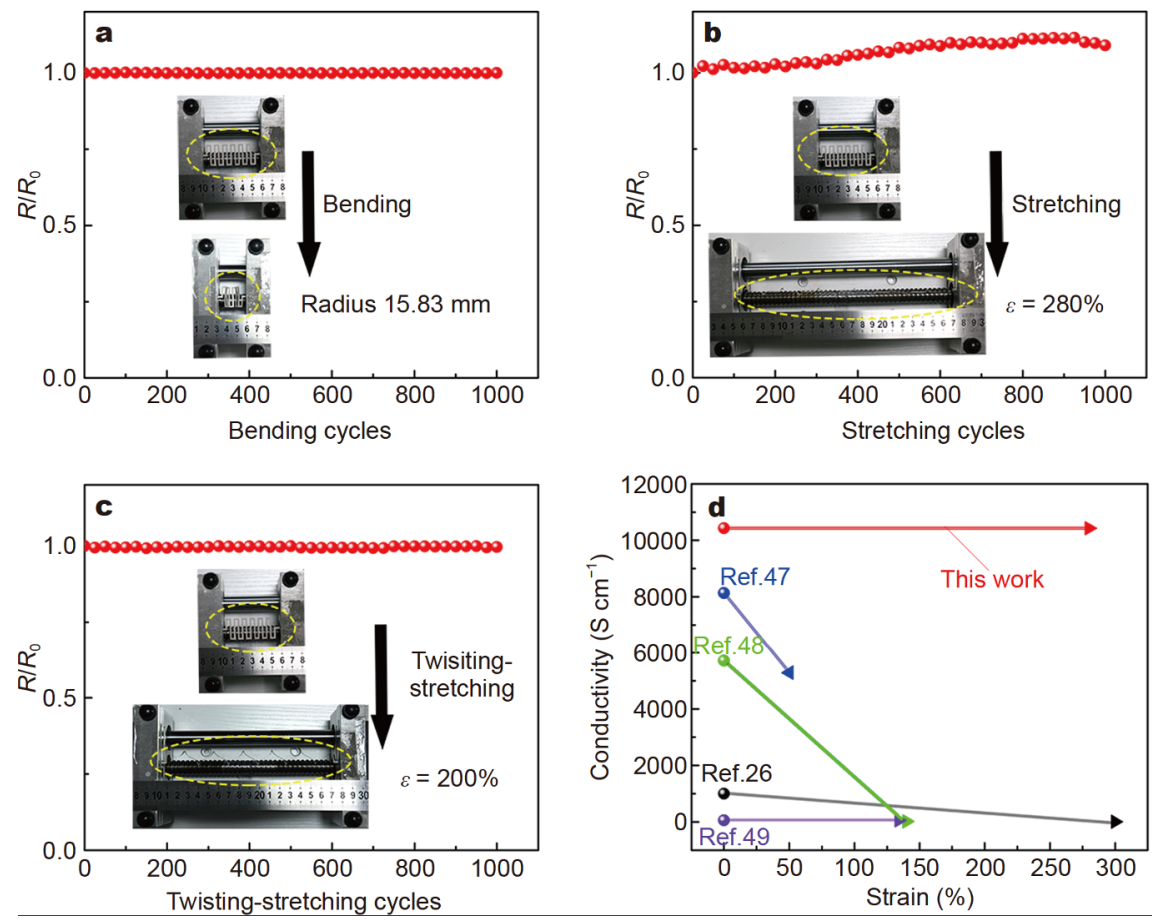

Figure 6 The relative resistance of the optimal interconnects as a function of (a) bending cycles, (b) stretching cycles, and (c) twisting-stretching cycles; (d) change of conductivity under stretching state. 


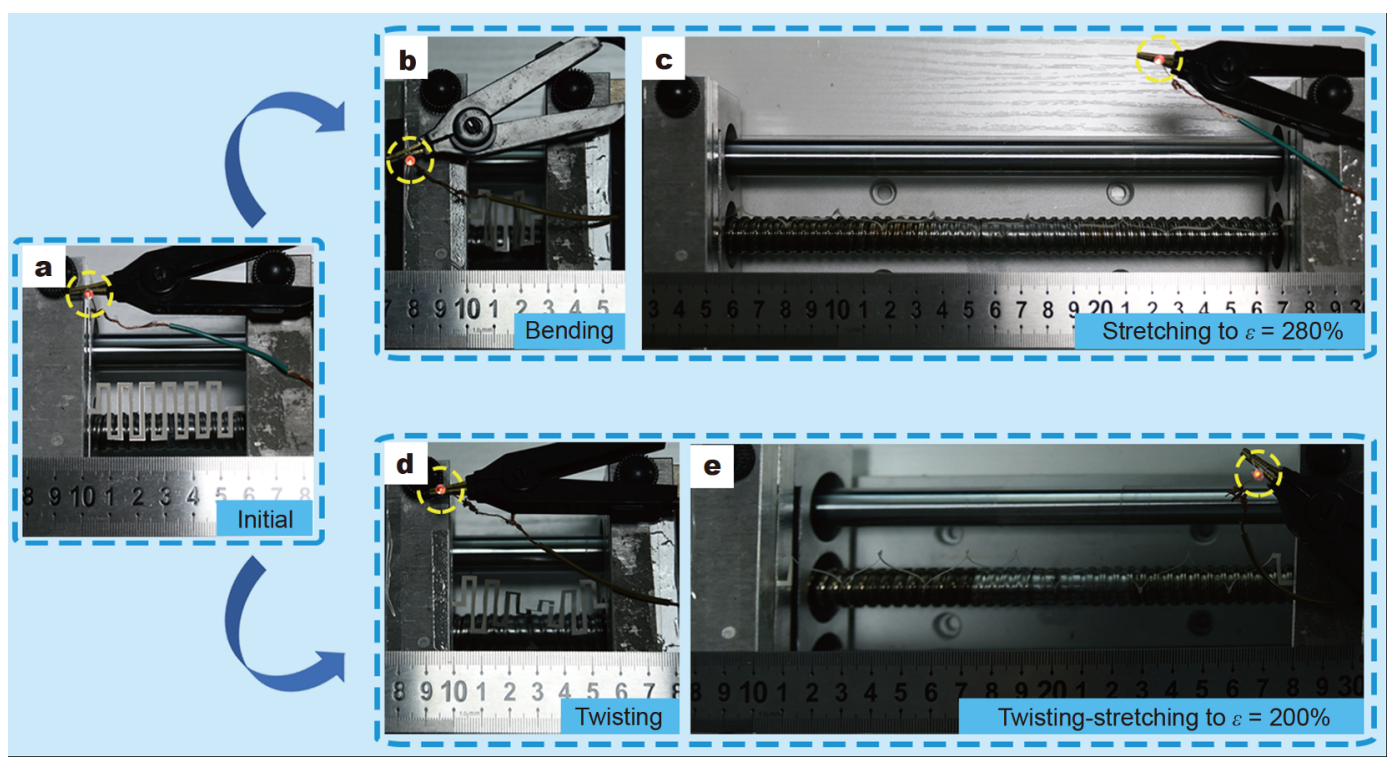

Figure 7 Stretchable flexible LED circuit based on interconnects under (a) initial, (b) bending, (c) stretching, (d) twisting and (e) twisting-stretching state.

spects in wearable electronics. All in all, we can foresee that these electrodes can be used as multi-function components, built into clothing, skin or wearable electronics, which will greatly affect people's electronic life.

\section{CONCLUSION}

In summary, the Ag NWs were obtained through a onepot solvothermal method. The water-based screen printing ink was formulated by combining the as-prepared $\mathrm{Ag}$ NWs solution with PVP addition agent. Additionally, the Ag NWs ink possesses viscosity of $94.51 \mathrm{~Pa}$ s when the shear rate is $0.1 \mathrm{~s}^{-1}$, simultaneously showing good rheological behavior during printing. The as-obtained $\mathrm{Ag}$ NWs ink was screen printed to PET through a topology designed screen plate, and then the topology designed interconnects were gained by kirigami method. The antistretching of interconnects was investigated by theoretical FEA calculations and experimental electrical tensile tests. Both results simultaneously showed that an increase of the wave number generated larger stretchability, and additionally the rectangular type of wave showed the best stretchability. Especially, the optimal interconnects based on 6 rectangular waves had the largest stretchability (302\%). Moreover, the interconnects possess outstanding mechanical reliability, so that the electric conductivity is almost unchanged at $\sim 10,427 \mathrm{~S} \mathrm{~cm}^{-1}$ after 1000 cycles of bending at 15.83 radius, stretching at $280 \%$ or twistingstretching at $200 \%$. The interconnects were successfully applied to stretchable flexible LED circuit. Thus, the in- terconnects reported here are promising for applications in the areas of stretchable electronics. In the future, we can use the die cutting to achieve large scale production, which is a process to use a machine for mass producing a single cut-out shape.

Received 17 April 2019; accepted 28 May 2019; published online 14 June 2019

1 Wu W. Inorganic nanomaterials for printed electronics: A review. Nanoscale, 2017, 9: 7342-7372

2 Wu W. Stretchable electronics: Functional materials, fabrication strategies and applications. Sci Tech Adv Mater, 2019, 20: 187-224

3 Tian B, Yao W, Zeng P, et al. All-printed, low-cost, tunable sensing range strain sensors based on $\mathrm{Ag}$ nanodendrite conductive inks for wearable electronics. J Mater Chem C, 2019, 7: 809-818

4 White MS, Kaltenbrunner M, Głowacki ED, et al. Ultrathin, highly flexible and stretchable pleds. Nat Photon, 2013, 7: 811-816

5 Sekitani T, Nakajima H, Maeda $\mathrm{H}$, et al. Stretchable active-matrix organic light-emitting diode display using printable elastic conductors. Nat Mater, 2009, 8: 494-499

6 Liang J, Li L, Niu X, et al. Elastomeric polymer light-emitting devices and displays. Nat Photon, 2013, 7: 817-824

7 Andersson P, Forchheimer R, Tehrani P, et al. Printable all-organic electrochromic active-matrix displays. Adv Funct Mater, 2007, 17: 3074-3082

8 Wei $\mathrm{Y}$, Chen $\mathrm{S}$, Lin $\mathrm{Y}$, et al. $\mathrm{Cu}-\mathrm{Ag}$ core-shell nanowires for electronic skin with a petal molded microstructure. J Mater Chem C, 2015, 3: 9594-9602

9 Vosgueritchian M, Tok JBH, Bao Z. Light-emitting electronic skin. Nat Photon, 2013, 7: 769-771

10 Matsuzaki R, Tabayashi K. Highly stretchable, global, and distributed local strain sensing line using GaInSn electrodes for 
wearable electronics. Adv Funct Mater, 2015, 25: 3806-3813

11 Lipomi DJ, Vosgueritchian M, Tee BCK, et al. Skin-like pressure and strain sensors based on transparent elastic films of carbon nanotubes. Nat Nanotech, 2011, 6: 788-792

$12 \mathrm{Gu} \mathrm{H}$, Zhang $\mathrm{H}, \mathrm{Ma} \mathrm{C}$, et al. Smart strain sensing organicinorganic hybrid hydrogels with nano barium ferrite as the crosslinker. J Mater Chem C, 2019, 7: 2353-2360

13 Wei $\mathrm{H}$, Wang $\mathrm{H}$, Xia $\mathrm{Y}$, et al. An overview of lead-free piezoelectric materials and devices. J Mater Chem C, 2018, 6: 12446-12467

14 Jiang D, Wang Y, Li B, et al. Flexible sandwich structural strain sensor based on silver nanowires decorated with self-healing substrate. Macromol Mater Eng, 2019, 143: 1900074

15 Jiang D, Murugadoss V, Wang Y, et al. Electromagnetic interference shielding polymers and nanocomposites-a review. Polym Rev, 2019, 59: 280-337

16 Wang C, Murugadoss V, Kong J, et al. Overview of carbon nanostructures and nanocomposites for electromagnetic wave shielding. Carbon, 2018, 140: 696-733

$17 \mathrm{Wu} \mathrm{N}, \mathrm{Xu} \mathrm{D}$, Wang $\mathrm{Z}$, et al. Achieving superior electromagnetic wave absorbers through the novel metal-organic frameworks derived magnetic porous carbon nanorods. Carbon, 2019, 145: 433444

18 Liang T, Qi L, Ma Z, et al. Experimental study on thermal expansion coefficient of composite multi-layered flaky gun propellants. Compos Part B-Eng, 2019, 166: 428-435

19 Rogers JA, Someya T, Huang Y. Materials and mechanics for stretchable electronics. Science, 2010, 327: 1603-1607

20 Zhang S, Li Y, Tian Q, et al. Highly conductive, flexible and stretchable conductors based on fractal silver nanostructures. J Mater Chem C, 2018, 6: 3999-4006

21 Guan YS, Zhang Z, Tang Y, et al. Kirigami-inspired nanoconfined polymer conducting nanosheets with $2000 \%$ stretchability. Adv Mater, 2000, 30: 1706390

22 Prasad O, Jha P, Pillai S, et al. Interconnects on elastomers: Optimizing for stretchability, speed and layout area. Flex Print Electron, 2017, 2: 045007

23 Yu C, Masarapu C, Rong J, et al. Stretchable supercapacitors based on buckled single-walled carbon-nanotube macrofilms. Adv Mater, 2009, 21: 4793-4797

24 Lacour SP, Wagner S, Huang Z, et al. Stretchable gold conductors on elastomeric substrates. Appl Phys Lett, 2003, 82: 2404-2406

25 Lacour SP, Jones J, Wagner S, et al. Stretchable interconnects for elastic electronic surfaces. Proc IEEE, 2005, 93: 1459-1467

26 Catenacci MJ, Reyes C, Cruz MA, et al. Stretchable conductive composites from $\mathrm{Cu}-\mathrm{Ag}$ nanowire felt. ACS Nano, 2018, 12: 36893698

27 Fan JA, Yeo WH, Su Y, et al. Fractal design concepts for stretchable electronics. Nat Commun, 2014, 5: 3266

28 Su Y, Wang S, Huang YA, et al. Elasticity of fractal inspired interconnects. Small, 2015, 11: 367-373

29 Dang W, Vinciguerra V, Lorenzelli L, et al. Printable stretchable interconnects. Flex Print Electron, 2017, 2: 013003

30 Jiang C, Zhong Z, Liu B, et al. Coffee-ring-free quantum dot thin film using inkjet printing from a mixed-solvent system on modified $\mathrm{ZnO}$ transport layer for light-emitting devices. ACS Appl Mater Interfaces, 2016, 8: 26162-26168

31 Choi MK, Yang J, Kang K, et al. Wearable red-green-blue quantum dot light-emitting diode array using high-resolution intaglio transfer printing. Nat Commun, 2015, 6: 7149

32 Fukuda K, Takeda Y, Yoshimura Y, et al. Fully-printed high-per- formance organic thin-film transistors and circuitry on onemicron-thick polymer films. Nat Commun, 2014, 5: 4147

33 Liang J, Tong K, Pei Q. A water-based silver-nanowire screen-print ink for the fabrication of stretchable conductors and wearable thinfilm transistors. Adv Mater, 2016, 28: 5986-5996

34 Cao R, Wang J, Zhao S, et al. Self-powered nanofiber-based screenprint triboelectric sensors for respiratory monitoring. Nano Res, 2018, 11: 3771-3779

35 Huang L, Huang Y, Liang J, et al. Graphene-based conducting inks for direct inkjet printing of flexible conductive patterns and their applications in electric circuits and chemical sensors. Nano Res, 2011, 4: 675-684

36 Lu Q, Liu L, Yang S, et al. Facile synthesis of amorphous FeOOH/ $\mathrm{MnO}_{2}$ composites as screen-printed electrode materials for allprinted solid-state flexible supercapacitors. J Power Sources, 2017, 361: $31-38$

37 Liu L, Lu Q, Yang S, et al. All-printed solid-state microsupercapacitors derived from self-template synthesis of Ag@PPy nanocomposites. Adv Mater Technol, 2018, 3: 1700206

38 Krebs FC, Jørgensen M, Norrman K, et al. A complete process for production of flexible large area polymer solar cells entirely using screen printing-first public demonstration. Sol Energy Mater Sol Cells, 2009, 93: 422-441

39 Singh M, Haverinen HM, Dhagat P, et al. Inkjet printing-process and its applications. Adv Mater, 2010, 22: 673-685

40 Yim C, Kockerbeck ZA, Jo SB, et al. Hybrid copper-silvergraphene nanoplatelet conductive inks on PDMS for oxidation resistance under intensive pulsed light. ACS Appl Mater Interfaces, 2017, 9: 37160-37165

41 Cui Z, Han Y, Huang Q, et al. Electrohydrodynamic printing of silver nanowires for flexible and stretchable electronics. Nanoscale, 2018, 10: 6806-6811

42 Yao W, Tian Q, Liu J, et al. Large-scale synthesis and screen printing of upconversion hexagonal-phase $\mathrm{NaYF}_{4}: \mathrm{Yb}^{3+}, \mathrm{Tm}^{3+} / \mathrm{Er}^{3+} /$ $\mathrm{Eu}^{3+}$ plates for security applications. J Mater Chem C, 2016, 4: 6327-6335

43 Kamyshny A, Magdassi S. Conductive nanomaterials for printed electronics. Small, 2014, 10: 3515-3535

44 Matsuhisa N, Kaltenbrunner M, Yokota T, et al. Printable elastic conductors with a high conductivity for electronic textile applications. Nat Commun, 2015, 6: 7461

45 Bandodkar AJ, Nuñez-Flores R, Jia W, et al. All-printed stretchable electrochemical devices. Adv Mater, 2015, 27: 3060-3065

46 Xue Q, Yao W, Liu J, et al. Facile synthesis of silver nanowires with different aspect ratios and used as high-performance flexible transparent electrodes. Nanoscale Res Lett, 2017, 12: 480

$47 \mathrm{Xu} \mathrm{F}, \mathrm{Zhu}$ Y. Highly conductive and stretchable silver nanowire conductors. Adv Mater, 2012, 24: 5117-5122

48 Chun KY, Oh Y, Rho J, et al. Highly conductive, printable and stretchable composite films of carbon nanotubes and silver. Nat Nanotech, 2010, 5: 853-857

49 Sekitani T, Noguchi Y, Hata K, et al. A rubberlike stretchable active matrix using elastic conductors. Science, 2008, 321: 1468-1472

Acknowledgements This work was supported by the National Natural Science Foundation of China (51471121), the Basic Research Plan Program of Shenzhen City (JCYJ20170303170426117), the Natural Science Foundation of Jiangsu Province (BK20160383), the Fundamental Research Funds for the Central Universities (2042018kf203) and Wuhan University. 
Author contributions Li X, Ruan X, Xia R and Wu W conceived the project and wrote the paper. $\mathrm{Li} \mathrm{X}$ and $\mathrm{Wu} \mathrm{W}$ designed the experiments. Li X performed the fabrication of the interconnects and measured the electrical and mechanical properties. Ruan X conducted the theoretical simulations via finite element analysis. Yao W, Liu L, Tian B, Wang $\mathrm{H}$ and Feng $\mathrm{Y}$ provided inputs into the design of the experiments and the preparation of the manuscript.

Conflict of interest The authors declare that they have no competing interests.

Supplementary information Supporting data are available in the online version of the paper.

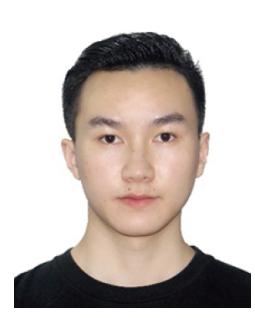

Xuan $\mathbf{L i}$ is currently pursuing a Master degree under the supervision of Prof. Wei Wu in the School of Printing and Packaging at Wuhan University. His current research focuses on the functional nanomaterials for printed electronics.

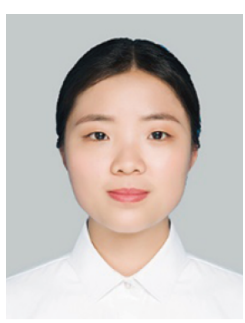

Xiaoli Ruan is currently pursuing a Master degree under the supervision of Prof. Re Xia in the School of Power and Mechanical Engineering at Wuhan University. Her current research focuses on the mechanical properties of the composite materials.

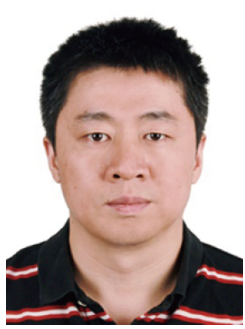

Re Xia received his $\mathrm{PhD}$ degree in 2010 from the Department of Engineering Mechanics, Tsinghua University, China. He has been an associate professor in the Department of Mechanical Engineering, School of Power and Mechanical Engineering, Wuhan University since 2011. He is currently the deputy director of the Key Laboratory of Hydraulic Machinery Transients (Wuhan University), Ministry of Education. His research interests include the mechanical properties of nanoporous materials and composite

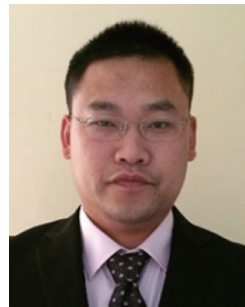

Wei $\mathbf{W u}$ received his $\mathrm{PhD}$ degree in 2011 from the Department of Physics, Wuhan University, China. He then joined the groups of Prof. Daiwen Pang at Wuhan University (2011) and Prof. V. A. L. Roy at the City University of Hong Kong (2014) as a postdoctoral fellow. Now he is the full professor and Director of the Laboratory of Printable Functional Nanomaterials and Printed Electronics, School of Printing and Packaging, Wuhan University. He has published more than 80 papers, which have received more than 4000 citations. He received the STAM Best Paper Award in 2017 and Hong Kong Scholars Award in 2014. He is also the topical editor of Frontiers in Materials. His research interests include the synthesis and applications of functional nanomaterials, printed electronics and intelligent packaging.

剪纸拓扑结构引发的大面积高可拉伸柔性互连电 极: 直接印刷法制备和力学机理

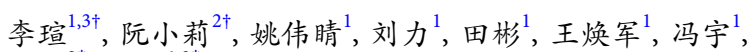
夏热 ${ }^{2 *}$, 吴伟 ${ }^{1,3^{*}}$

摘要 结合拓扑设计和印刷方法来实现高可拉伸性互连电极的制 备有望成为一种非常有前途的策略. 本文借助丝网印刷技术, 所配 制的银纳米线水性油墨被用于制备大面积(超过 $335 \mathrm{~mm} \times 177 \mathrm{~mm}$ ) 的高可拉伸柔性互连电极. 电极的可拉伸性通过引入剪纸拓扑结 构来实现, 并通过有限元法分析了所设计的系列剪纸拓扑模型对 变形顺应性的影响, 以此探究其最优拉伸性. 系统的力学机理分析 表明结构的可拉伸性随着波数的增加而增加, 与锯齿形波和正弦 形波剪纸结构相比, 矩形波结构具有最佳可拉伸性. 此外, 电极的 电学性能测试结果与有限元分析预测的结果相一致. 该方法制备 的互连电极具有 $10427 \mathrm{~S} \mathrm{~cm}^{-1}$ 的高电导率, 并且表现出优异的力 学稳定性, 经过 1000 次的弯曲循环(弯曲半径为 $15.83 \mathrm{~mm}$ )、拉伸 循环(拉伸率为 $280 \%$ ) 和扭转-拉伸循环 (扭转 $180^{\circ}$, 拉伸率为 $200 \%$ ) 测试, 电导率始终保持稳定. 基于拓扑设计的互连电极被应用于可 拉伸柔性LED电路, 进一步证明其在下一代可拉伸电子产品中具有 广泛的应用前景.

structures. 